ARTICLE

Received 8 Dec 2014 | Accepted 17 Apr 2015 | Published 26 May 2015

DOl: $10.1038 /$ ncomms8210

OPEN

\title{
An incompressible state of a photo-excited electron gas
}

\author{
Alexei D. Chepelianskii ${ }^{1}{ }^{2}$, Masamitsu Watanabe ${ }^{3}$, Kostyantyn Nasyedkin ${ }^{4}$, Kimitoshi Kono ${ }^{4,5,6}$ \\ $\&$ Denis Konstantinov ${ }^{7}$
}

Two-dimensional electrons in a magnetic field can form new states of matter characterized by topological properties and strong electronic correlations as displayed in the integer and fractional quantum Hall states. In these states, the electron liquid displays several spectacular characteristics, which manifest themselves in transport experiments with the quantization of the Hall resistance and a vanishing longitudinal conductivity or in thermodynamic equilibrium when the electron fluid becomes incompressible. Several experiments have reported that dissipationless transport can be achieved even at weak, non-quantizing magnetic fields when the electrons absorb photons at specific energies related to their cyclotron frequency. Here we perform compressibility measurements on electrons on liquid helium demonstrating the formation of an incompressible electronic state under these resonant excitation conditions. This new state provides a striking example of irradiation-induced self-organization in a quantum system.

\footnotetext{
${ }^{1}$ LPS, Université Paris-Sud, CNRS, UMR 8502, Orsay F-91405, France. ${ }^{2}$ Cavendish Laboratory, University of Cambridge, J J Thomson Avenue, Cambridge CB3 OHE, UK. ${ }^{3}$ Low Temperature Physics Laboratory, RIKEN, Wako, Saitama 351-0198, Japan. ${ }^{4}$ Quantum Condensed Phases Research Team, RIKEN CEMS, Wako, Saitama 351-0198, Japan. ${ }^{5}$ Institute of Physics, National Chiao Tung University, Hsinchu 30010, Taiwan. ${ }^{6}$ Institute of Physics, Kazan Federal University, Kazan 420008, Russia. ${ }^{7}$ Okinawa Institute of Science and Technology, Onna, Okinawa 904-0412, Japan. Correspondence and requests for materials should be addressed to A.C. (email: alexei.chepelianskii@u-psud.fr).
} 
T he discovery of the integer and fractional quantum Hall effects $^{1-6}$ revealed the existence of new states of matter characterized by topological properties and strong electronic correlations triggering an intense theoretical and experimental research activity. These efforts lead to a detailed microscopic understanding of the main experimental phenomena and to some of the most beautiful conceptual breakthroughs in condensed matter physics ${ }^{7}$. The observation of a new dissipationless transport regime at low magnetic fields under microwave irradiation ${ }^{8,9}$ raised a new challenge regarding our understanding of two-dimensional electron systems. Microwaveinduced zero-resistance states (ZRSs) appear at high-microwave excitation powers when the ratio between the photon energy $\hbar \omega$ and Landau level spacing $\hbar \omega_{\mathrm{c}}$ is close to a value within the fraction series: $J=\omega / \omega_{\mathrm{c}}=1+1 / 4,2+1 / 4, \ldots$ In theory, however, the motion of electrons in a magnetic field in ultra-clean samples is well described as a harmonic oscillator where the selection rules only allow transitions between nearby oscillator states corresponding to $J=1$. The theoretical explanations proposed, so far, have attempted to resolve this contradiction by considering the role of sharp inhomogeneities due to a short-range disorder potential $^{10-12}$, edges ${ }^{13,14}$ and contacts ${ }^{15}$. The dominant microscopic picture for ZRS is currently an ensemble of domains with vanishing local conductivity ${ }^{12}$ but the formation of a collective state with long-range order has also been suggested ${ }^{16,17}$. So far, the experimental evidence does not provide a definite proof in support of one of the available models and despite intense experimental efforts ${ }^{18-24}$ the microscopic nature of ZRS remains a puzzle. One of the difficulties is that while the manifestations of ZRS in transport phenomena are spectacular, such as vanishing longitudinal resistance, other indications of these novel electronic states remain elusive.

The observation of ZRS for surface electrons on helium under intersubband excitation ${ }^{25,26}$ has opened a new research direction in this field since the strong Coulomb interactions in this system allow collective effects, such as, for example, Wigner crystallization, to be observed more readily ${ }^{27,28}$. Previously, we reported a strong redistribution of the electron density under irradiation that coincided with the appearance of ZRS ${ }^{29}$, although

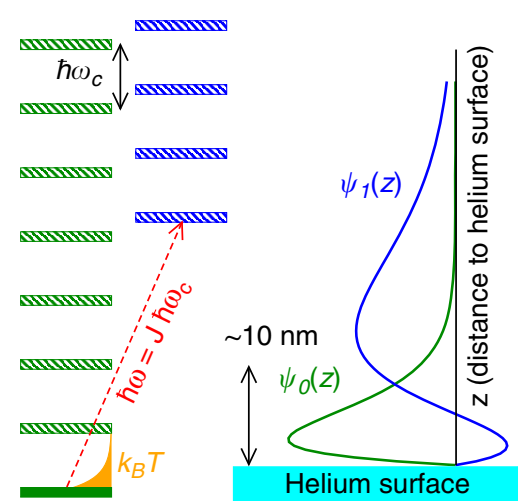

Figure 1 | Accessible energy levels. Level diagram of the accessible states for the surface electrons in our experiment. Several bound states are formed owing to the attractive image charge potential created by the helium surface, the ground state and the first excited state are separated by energy $\hbar \omega$. Free motion along the helium surface transforms these states into conduction bands which under a quantizing magnetic field split into a manifold of discrete Landau levels. The ground state manifold is characterized by a wavefunction $\psi_{0}(z)$ that is localized close to the helium surface, whereas the wavefunction of the excited state manifold is centered farther away from the helium surface. the underlying mechanism was not elucidated. Since the formation of ZRS in a Hall system coincides with vanishing conductivity, the observed redistribution may simply be a consequence of the expected long charge relaxation rates in this regime. In the experiments presented here, we systematically study the behaviour of the electronic density under irradiation and demonstrate a regime in which electrons stabilize at a fixed steady-state density independent of their initial density profile and the electrostatic confinement potential. Since in this regime the electron density is not changed by an increase of the holding electrostatic forces, which tend to compress the electron cloud, we describe this new phase of the electron gas as an incompressible state.

\section{Results}

Description of the system. A ensemble of electrons was trapped on a liquid helium surface forming a non-degenerate twodimensional electron gas. The energy levels accessible to the surface electrons in a quantizing perpendicular magnetic field are shown in Fig. 1. They are formed by Landau levels separated by energy $\hbar \omega_{c}$ and intersubband excitations of energy $\hbar \omega$ perpendicular to the helium surface ${ }^{27,28}$. Our experiments are performed at a temperature of $T=300 \mathrm{mK}$ much smaller than the Landau level spacing $k_{\mathrm{B}} T \ll \hbar \omega_{\mathrm{c}}$ so that in equilibrium the electrons mainly fill the lowest Landau level, whereas under resonant irradiation at energy $\hbar \omega$, they can be excited into another subband manifold ${ }^{25,26}$. Note that the two-level system formed by the two subbands has been proposed as a candidate system for quantum computing ${ }^{30}$. Spatially, the electrons are distributed between the two regions on the helium surface (see Fig. 2), a central region above the disc-shaped electrode at potential $V_{\mathrm{d}}$ and a surrounding guard region above the ring electrode held at potential $V_{\mathrm{g}}$. We denote $n_{\mathrm{e}}$ and $n_{\mathrm{g}}$ as the mean electron densities in the central and guard regions, respectively. As in a field effect transistor, $n_{\mathrm{e}}$ and $n_{\mathrm{g}}$ can be controlled by changing the potentials $V_{\mathrm{d}}$ and $V_{\mathrm{g}}$. The key difference here is that for surface electrons the

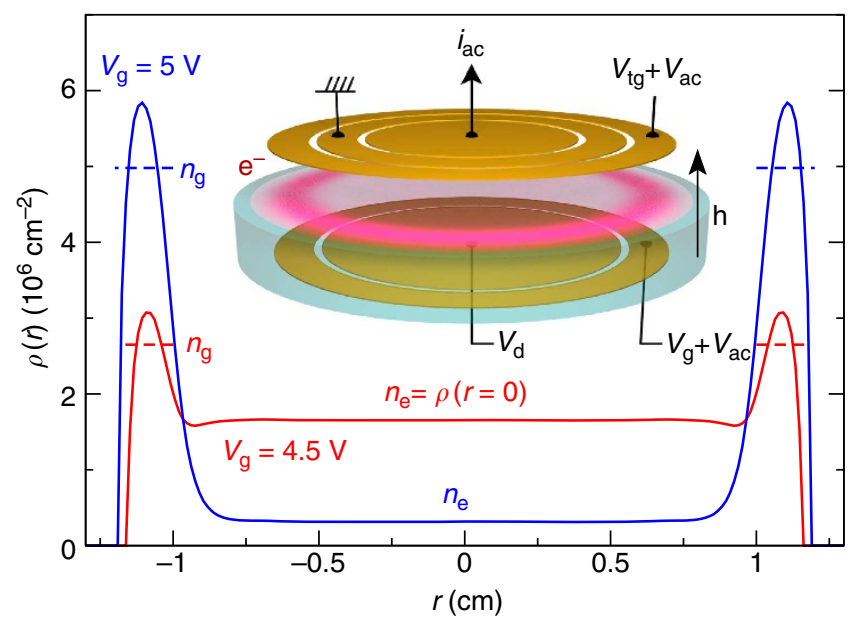

Figure 2 | Electron distribution inside the cell. Theoretical electron density profiles $\rho(r)$ for different values of the guard potential $V_{g}$ for a cloud with $N_{\mathrm{e}}=8 \times 10^{6}$ trapped electrons and corresponding values of the mean electron density in the centre $n_{\mathrm{e}}$ and in the guard $n_{\mathrm{g}}$. The inset illustrates the helium cell geometry in our experiment and the electrostatic potentials applied to the electrodes during the compressibility experiments. The top of the cell is split in three circular electrodes with radii $R_{\mathrm{i}}=0.7, R_{\mathrm{d}}=1$, $R_{\mathrm{g}}=1.3 \mathrm{~cm}$, and the height of the cell is $h=2.6 \mathrm{~mm}$. For compressibility measurements, an a.c. driving potential is applied to both top and bottom guard electrodes and the induced image charge current $i_{\text {a.c. }}$ is measured on the central top electrode. 
total number of electrons $N_{e}$ in the cloud is fixed as long as the positive potentials $V_{\mathrm{d}}$ and $V_{\mathrm{g}}$ are sufficiently strong to balance the electron-electron Coulomb repulsion. Examples of simulated electron density profiles for different values of $V_{\mathrm{g}}$ are shown in Fig. 2, the simulations were performed within an electrostatic model as described in refs 31-33.

In addition to controlling the density profile of the electron cloud $\rho(r)$, the potentials $V_{\mathrm{d}}$ and $V_{\mathrm{g}}$ also change the perpendicular holding field in the cell $E_{z}$. To avoid this unwanted effect in our experiments, we fixed the value of $V_{\mathrm{d}}$ and changed the potential $V_{\mathrm{tg}}$ simultaneously with $V_{\mathrm{g}}$ keeping the difference $V_{\mathrm{tg}}-V_{\mathrm{g}}$ between top and bottom guard electrode voltages equal to $V_{\mathrm{d}}$. This choice ensures a uniform value of $E_{z}$ across the cell. Since lowering the potential $V_{\mathrm{g}}$ compresses the electron cloud towards the centre of the helium cell, we define the compressibility of the electron system as $\chi=-d n_{\mathrm{e}} / d V_{\mathrm{g}}$. In this definition, the electrostatic potential plays the role of the chemical potential in quantum Hall systems. This difference is due to the nondegenerate statistics for electrons on helium for which the Fermi energy is much smaller than the thermal energy.

Compressibility in equilibrium. We developed the following method to measure the compressibility of the electron cloud. An a.c. voltage excitation with amplitude $V_{\text {a.c. }}=25 \mathrm{~m} V$ was applied on the top and bottom guard electrodes (see Fig. 2) at a low frequency $f_{\text {a.c. }} \simeq 2 \mathrm{~Hz}$, for which the electron density quasistatically follows the driving potential. The induced modulation of $n_{\mathrm{e}}$ was measured by recording the a.c. current $i_{\text {a.c. }}$ created by the motion of image charges on the top central electrode with radius $R_{\mathrm{i}}=0.7 \mathrm{~cm}$. The correspondence between the variation of $n_{\mathrm{e}}$ and $i_{\text {a.c. }}$ was established using plane capacitance electrostatics, for which an electron trapped at the middle of the cell induces half an image charge of $e / 2$ on the top electrode. The use of a simplified electrostatic model is justified here, since the gradients of $\rho(r)$ are located away from the electrode on which $i_{\text {a.c. }}$ is measured. This leads to the following expression for the compressibility $\chi=-d n_{\mathrm{e}} / d V_{\mathrm{g}}$ :

$$
\chi=\frac{i_{\text {a.c. }}}{e \pi^{2} f_{\text {a.c. }} R_{\mathrm{i}}^{2} V_{\text {a.c. }}} .
$$

The presented measurement technique has a strong similarity to that used in compressibility experiments in the quantum Hall regime $^{34}$, providing an additional justification for our definition of compressibility. In Supplementary Note 1, we provide a more thorough discussion on our definition of compressibility and give a detailed derivation of Equation 1, which is explained in Supplementary Note 2.

Using Equation 1, the dependence $n_{\mathrm{e}}\left(V_{\mathrm{g}}\right)$ can be reconstructed by integrating $\chi$ with respect to $V_{\mathrm{g}}$ starting from the high $V_{\mathrm{g}}$ limit where $n_{\mathrm{e}}=0$. The obtained results are illustrated in Fig. 3, which shows $i_{\text {a.c. }}$ and the corresponding density $n_{\mathrm{e}}$ as a function of $V_{\mathrm{g}}$ for several $N_{\mathrm{e}}$ values. The experimental curves for $n_{\mathrm{e}}\left(V_{\mathrm{g}}\right)$ are compared with the results of electrostatic simulations ${ }^{31-33,35}$ that use the total electron number $N_{\mathrm{e}}$ as the single fitting parameter for each of the obtained curves. The simulations exhibit an extremely good agreement with the experimental results. The dependence $n_{\mathrm{e}}\left(V_{\mathrm{g}}\right)$ can be understood as follows: a large positive potential $V_{\mathrm{g}}$ attracts the electrons towards the guard electrodes, whereas at low $V_{\mathrm{g}}$ the electrons are repelled from the guard region and concentrate at the centre of the cell. The potential from the guard electrodes is then almost completely screened, leading to a value of $n_{\mathrm{e}}$ that is almost independent of $V_{\mathrm{g}}$. At intermediate $V_{g}$, electrons occupy both the central and guard regions. In this case, $n_{\mathrm{e}}$ decreases linearly with increasing $V_{\mathrm{g}}$ until the central region becomes completely depleted. At this point,

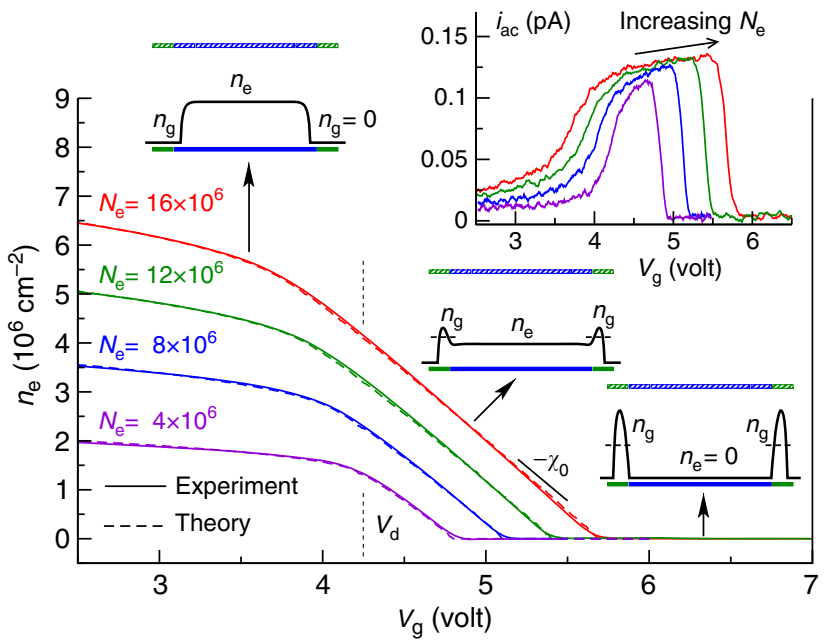

Figure 3 | Density without irradiation. Dependence of $n_{e}$ on the guard potential $V_{\mathrm{g}}$ without irradiation obtained using Equation 1 for different numbers of trapped electrons $N_{\mathrm{e}}$, the experimental values for the current are shown in the inset. The total number of electrons in the cloud is determined by comparing the measured dependence $n_{e}\left(V_{g}\right)$ with theoretical calculations based on electrostatic modelling of the electron cloud. The sketches illustrate the typical electron density profiles along the $n_{\mathrm{e}}\left(V_{\mathrm{g}}\right)$ curves. A very good agreement between experiment and modelling is achieved using the total electron number $N_{e}$ as the only fitting parameter. Over a large range of $V_{\mathrm{g}}$ and $N_{\mathrm{e}}$, the compressibility is well approximated by the constant value $\chi_{0} \simeq 2.9 \times 10^{6} \mathrm{~cm}^{-2} \mathrm{~V}^{-1}$. Temperature was

$T=300 \mathrm{mK}$, we verified that as expected these measurements were independent of the magnetic field.

$n_{\mathrm{e}}=0$ regardless of the value of $V_{\mathrm{g}}$. In the intermediate regime, the compressibility $\chi=-\left(d n_{\mathrm{e}}\right) /\left(d V_{\mathrm{g}}\right)$ depends only weakly on the total number of trapped electrons $N_{\mathrm{e}}$ and the values of the confining potentials. This observation can be understood by considering a simplified electrostatic model in which the two electron reservoirs in the disc and guard are treated as plane capacitors. This model, which is presented in more detail in Supplementary Note 3 , leads to a value for $\chi$ that depends only on the geometrical cell parameters (provided the disc/guard reservoirs are not empty):

$$
\chi_{0}=-\frac{d n_{\mathrm{e}}}{d V_{\mathrm{g}}}=\frac{4 \epsilon_{0}}{e h\left(1+S_{\mathrm{d}} / S_{\mathrm{g}}\right)}
$$

Here, $h=2.6 \mathrm{~mm}$ is the cell height, $S_{\mathrm{d}}=\pi R_{\mathrm{d}}^{2}$ and $S_{\mathrm{g}}=$ $\pi\left(R_{\mathrm{g}}^{2}-R_{\mathrm{d}}^{2}\right)$ are the surface areas of the bottom disc/guard electrodes $\left(R_{\mathrm{g}}\right.$ being the outer radius of the guard electrodes) and $\epsilon_{0}$ is the vacuum permittivity. We experimentally find $\chi_{0} \simeq 2.9 \times 10^{6} \mathrm{~cm}^{-2} \mathrm{~V}^{-1}$ in good agreement with the estimation obtained using the geometrical cell parameters $R_{\mathrm{d}}=1 \mathrm{~cm}$ and $R_{\mathrm{g}}=1.3 \mathrm{~cm}$. This reference value will be used to normalize the compressibility in our following experiments.

Compressibility under irradiation. We next present our compressibility measurements in the presence of microwave irradiation, focusing on the ZRS fraction $J=\omega / \omega_{\mathrm{c}}=6.25$, with $\omega=2 \pi \times 139 \mathrm{GHz}$ and a magnetic field of $B=0.79 \mathrm{~T}$. Outside ZRS regions the compressibility is not changed by microwave irradiation since it is independent of the conductivity of the system $\sigma_{\mathrm{xx}}$ provided it remains finite. The holding field, identical in the central and guard regions $\left(V_{\mathrm{d}}=V_{\mathrm{g}}-V_{\mathrm{tg}}=4.24 \mathrm{~V}\right)$, was chosen to tune the photon energy in resonance with the intersubband transition. 
a

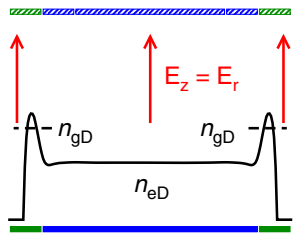

C

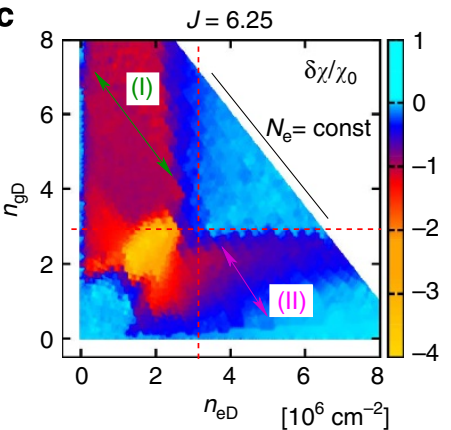

d

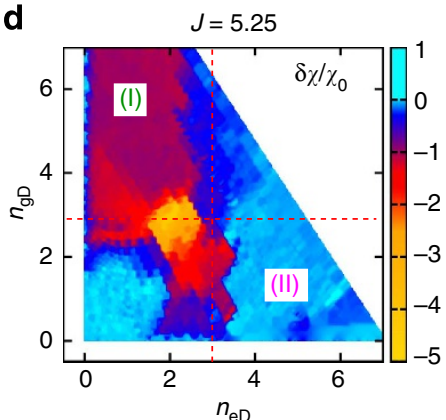

b

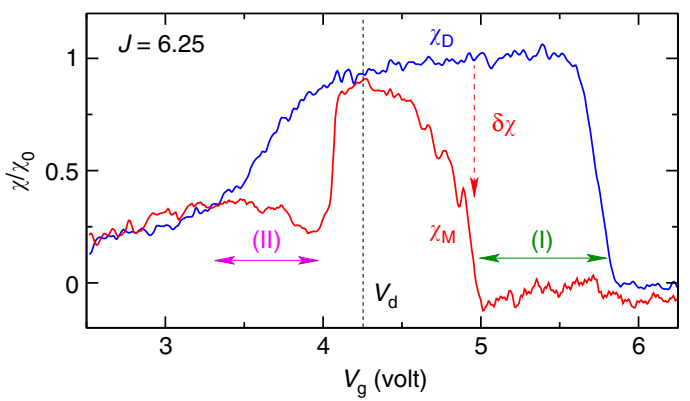

e

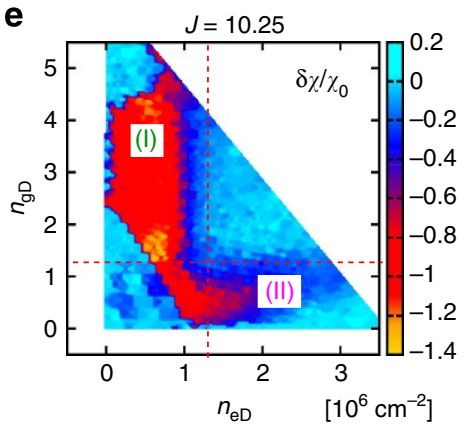

Figure 4 | Compressibility under irradiation. Compressibility $\chi$ as a function of guard voltage $V_{\mathrm{g}}$ in the dark $\left(\chi_{\mathrm{d}}\right)$ and under microwave irradiation $\left(\chi_{M}\right)$ at a frequency of $\omega=2 \pi \times 139 \mathrm{GHz}$ and a magnetic field $B=0.79$ T corresponding to $J=\omega / \omega_{\mathrm{c}}=6.25$. As shown in (a), the perpendicular electric field was fixed to the intersubband resonance value $E_{r}$ in both the central and guard regions. (b) The compressibility under microwave irradiation for $N_{\mathrm{e}}=18 \times 10^{6}$, $\chi$ changes under irradiation in two distinct regions: region (I), where $\chi_{M}$ almost vanishes under irradiation and region (II), where $\chi_{M}$ is significantly reduced. (c) $\delta \chi / \chi_{0}$ measured at different $N_{\mathrm{e}}$ values as a function of the equilibrium density at the centre $n_{\mathrm{eD}}$ and in the guard $n_{\mathrm{gD}}$. The boundaries of the anomalous regions (I) and (II) are aligned along lines of constant density $n_{\mathrm{eD}}$ and $n_{\mathrm{gD}}$, respectively. The colour scale indicates the change in the compressibility under irradiation, the value $\delta / / \chi_{0}=-1$ (red colour) corresponding to incompressible states. The positions of the coloured arrows corresponds to the trace in $\mathbf{b}$. $(\mathbf{d}, \mathbf{e})$ Results similar to $\mathbf{c}$ but obtained in two other ZRS regions: $J=5.25$ and $J=10.25$. The vertical/horizontal boundaries coincide approximately at $J=5.25$ and $J=6.25$ but are located at significantly lower densities for $J=10.25$.

The compressibilities measured in the dark $\chi_{\mathrm{d}}$ and under irradiation $\chi_{M}$ are compared in Fig. 4. Two singular regions where $\chi_{M}$ and $\chi_{\mathrm{d}}$ differ are present: region (I) at high $V_{\mathrm{g}}$ and region (II) at low $V_{\mathrm{g}}$. Hereafter on we use different notations for the average densities in the dark $n_{\mathrm{eD}}$ and $n_{\mathrm{gD}}$ and under microwave irradiation $n_{\mathrm{eM}}$ and $n_{\mathrm{gM}}$ to avoid ambiguity. In region $(I)$, the dark density in the guard is higher than at the centre $n_{\mathrm{gD}}>n_{\mathrm{eD}}$, whereas in region (II) we have $n_{\mathrm{gD}}<n_{\mathrm{eD}}$. In both regions, a strong suppression of the compressibility is observed and $\chi_{M}$ strikingly vanishes in most of region $(I)$. In contrast, at $V_{\mathrm{g}}=V_{\mathrm{d}}=4.24 \mathrm{~V}$ where the electrons are distributed evenly between centre and the guard, the compressibility remains almost unchanged under irradiation: $\chi_{M} \simeq \chi_{\mathrm{d}} \simeq \chi_{0}$.

To clarify the physical origin of the anomalous regions $(I)$ and (II), we convert the experimentally controlled variables $V_{\mathrm{g}}$ and $N_{\mathrm{e}}$ to physically more relevant densities $n_{\mathrm{eD}}$ and $n_{\mathrm{gD}}$. We obtained $n_{\mathrm{eD}}$ and $N_{\mathrm{e}}$ from the compressibility measurements in the dark (as in Fig. 3). The quantity $n_{\mathrm{gD}}$ could not be measured directly in a reliable manner owing to the unavoidable effect of the density gradients in the guard region. We, thus, calculated $n_{\mathrm{gD}}$ by averaging the simulated density profiles $\rho(r)$ over the guard region. This procedure is justified by the excellent agreement between the compressibility measurements and our numerical simulations that we demonstrated in absence of irradiation.

Using this method, we summarize on the $n_{\mathrm{eD}}, n_{\mathrm{gD}}$ plane the changes in compressibility $\delta \chi=\chi_{M}-\chi_{\mathrm{d}}$ under irradiation measured at different values of $V_{\mathrm{g}}$ and $N_{\mathrm{e}}$ while fixing all the other parameters (magnetic field, microwave frequency and power, temperature and perpendicular electric field; the dependence on microwave power and magnetic field is shown in Supplementary
Figs 1-5). The results are shown in the colour-scale panels in Fig. 4. The anomalous regions (I) and (II) are upper bounded by lines of constant density in the centre $n_{\mathrm{eD}}=n_{\mathrm{c}}$ and in the guard $n_{\mathrm{gD}}=n_{\mathrm{c}}$ where we introduced $n_{\mathrm{c}} \simeq 3 \times 10^{6} \mathrm{~cm}^{-2}$. Indeed, for $n_{\mathrm{gD}}>n_{\mathrm{c}}$ and $n_{\mathrm{eD}}>n_{\mathrm{c}}$ the change in compressibility $|\delta \chi| \ll \chi_{0}$ is negligible and the electron density is still described by the electrostatics of the gas phase. Similarly, at low densities: $n_{\mathrm{eD}}$ and $n_{\mathrm{gD}}<1.5 \times 10^{6} \mathrm{~cm}^{-2}$, we also find no deviations from the equilibrium $\chi$ values. The incompressible regions (I) and (II) occupy the space $n_{\mathrm{eD}}<n_{\mathrm{c}}<n_{\mathrm{gD}}$ and a fraction of the space $n_{\mathrm{gD}}<n_{\mathrm{c}}<n_{\mathrm{eD}}$, they are characterized by $\delta \chi / \chi_{0} \simeq-1$ in Fig. 4 . Finally, in the remaining area on the $n_{\mathrm{eD}}, n_{\mathrm{gD}}$ plane, $\chi$ becomes negative under irradiation, for example, at $n_{\mathrm{eD}}=n_{\mathrm{gD}}=2 \times 10^{6} \mathrm{~cm}^{-2}$. To highlight the robustness of our results, Fig. 4 also shows similar data obtained at two other ZRS fractions: $J=5.25$ and $J=10.25$. Incompressible regions appear for these cases as well, we note that for $J=10.25$ the position of the boundary $n_{\mathrm{c}}$ is displaced towards significantly lower densities $n_{\mathrm{c}} \simeq 1.3 \times 10^{6} \mathrm{~cm}^{-2}$.

The incompressible regions with vanishing $\chi$ correspond to an unexpected regime where the density becomes independent of the compressing confinement potential $V_{\mathrm{g}}$. In the integer quantum Hall effect, incompressible phases appear owing to the finite energy required to add electrons to a system in which the Landau levels available at the Fermi energy are all fully occupied. This explanation, however, is not applicable to electrons on helium since they form a non-degenerate electron gas. Experiments on the quantum Hall effect have also shown that a vanishing longitudinal conductivity $\sigma_{\mathrm{xx}}$, can freeze a non-equilibrium electron density distribution since the charge relaxation time 
scales can become exponentially large ${ }^{36,37}$. An explanation based on the vanishing conductivity $\sigma_{\mathrm{xx}}$ seems natural owning to the coincidence between the onset of charge redistribution and ZRS. We can determine experimentally whether the incompressible behaviour can be explained only on the basis of $\sigma_{\mathrm{xx}}=0$. Indeed, a state with $\sigma_{\mathrm{xx}}=0$ is expected to freeze the existing density distribution due to the very long charge relaxation rates; thus, the final state should depend in a non-trivial way on the equilibrium density profile and on the kinetics of the transition to ZRS.

We developed the following approach to determine the central density under irradiation $n_{\mathrm{eM}}$ for different initial densities $n_{\mathrm{eD}}$. We performed compressibility measurements without irradiation as described in Fig. 3 to obtain $n_{\mathrm{eD}}$ as function of $V_{\mathrm{g}}$ at a fixed $N_{\mathrm{e}}$. Then, fixing $V_{\mathrm{g}}$, we irradiated the electron system with on/off pulses of millimetre waves creating a periodic displacement of the electron density $\delta n_{\mathrm{e}}=n_{\mathrm{eM}}-n_{\mathrm{eD}}$. This displacement induces a transient current of image charges on the measuring electrode $i_{\mathrm{pv}}(t)$, which within a plane capacitor approximation is related to the change in the electron density $\delta n_{\mathrm{e}}$ through the relation:

$$
\delta n_{\mathrm{e}}=n_{\mathrm{eM}}-n_{\mathrm{eD}}=\frac{2}{e \pi R_{\mathrm{i}}^{2}} \int i_{\mathrm{pv}}(t) d t
$$

The integral in this equation is evaluated over the time interval where the irradiation is switched off (a derivation of this relation is provided in Supplementary Note 4). Combining $\delta n_{\mathrm{e}}$ with the known values for $n_{\mathrm{eD}}$, we reconstructed the dependence of $n_{\mathrm{eM}}$ on the guard potential $V_{\mathrm{g}}$ : the results are shown oi Fig. 5 for several $N_{\mathrm{e}}$ values.

Deviations from $n_{\mathrm{eD}}$ mainly appear in two voltage regions, which are in good correspondence with the regions (I) and (II) outlined in Fig. 4. In region $(I)$, the density under irradiation exhibits a striking plateau as a function of $V_{\mathrm{g}}$ with a plateau density independent of $N_{\mathrm{e}}$. We emphasize that this plateau appears because of the cancellation between the decrease of $n_{\mathrm{eD}}$ at higher $V_{\mathrm{g}}$ and the increase of the area below $i_{\mathrm{pv}}(t)$ curves (see Fig. 5c and Equation 3), it is thus a highly non-trivial experimental result. These observations confirm the existence of an incompressible phase for electrons on helium and appear to exclude an explanation based only on $\sigma_{\mathrm{xx}}=0$ since the final state density does not depend on the density distribution in equilibrium for a wide range of parameters. Instead, our experiments imply the existence of a dynamical mechanism that stabilize the electron density to a fixed value.

We next comment on the sign of $\delta n_{\mathrm{e}}$. In region (I), the electrons migrate from the guard, where the densities are higher, to the centre of the electron cloud, whereas in region (II), the trend is opposite and electrons flow from the centre towards the edges of the electron cloud. In the latter region, narrow density plateaux are also observed, however, the plateau density value depends on $N_{\mathrm{e}}$ in contrast to region (I). An approximate calculation of $n_{\mathrm{gM}}$ shown in Fig. 5 suggests that in region (II), the transition to an incompressible state occurs owning to the pinning of the density inside the guard region. Thus, in the incompressible phase, the electron cloud transfers electrons from a high-density reservoir region, increasing the density in the low-density regions up to a plateau value.

\section{Discussion}

Since the formation of a non-equilibrium density profile increases the electron-electron repulsion energy, it is important to estimate the associated energy cost. Using the simplified electrostatic model (see Supplementary Note 5 for a detailed derivation), we find that the energy cost of the redistribution per electron $\Delta_{\mathrm{e}}$ is approximately:

$$
\Delta_{\mathrm{e}}=\frac{e S_{\mathrm{d}}}{2 \chi_{0} N_{\mathrm{e}}} \delta n_{\mathrm{e}}^{2}
$$

From the experimental values of $\delta n_{\mathrm{e}}=1.5 \times 10^{6} \mathrm{~cm}^{-2}$ and $N_{\mathrm{e}}=12.4 \times 10^{6}$ (estimated at point $V_{2}$ in Fig. 5), we find $\Delta_{\mathrm{e}} \simeq 0.1 \mathrm{eV}$. The presence of this large electrostatic barrier can explain why the incompressible regions occupy a narrower $V_{\mathrm{g}}$ range in the photocurrent data than in the compressibility measurement: for example, region $(I)$ has a width at least $0.6 \mathrm{~V}$ in Fig. 4 but a width of only $0.3 \mathrm{~V}$ in Fig. 5 . The main difference a

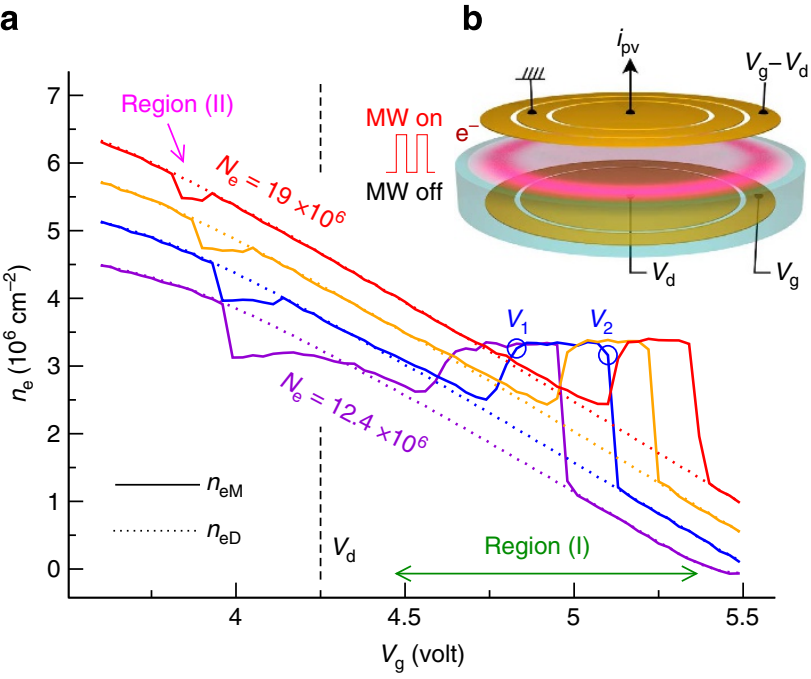

c

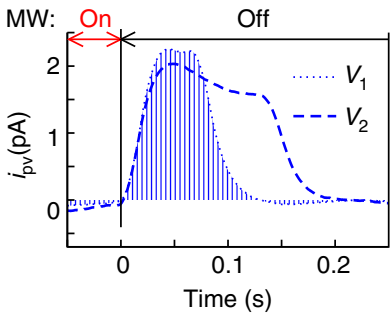

d

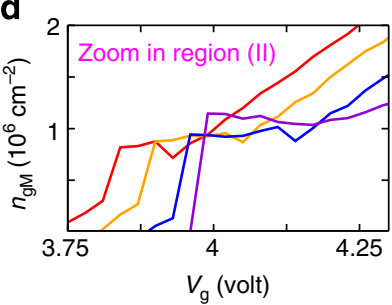

Figure 5 | Photocurrent density measurements. (a) Density under irradiation as determined from transient photocurrent measurements. The electron cloud was prepared at different initial density distributions by changing $V_{g}$ and performing experiments at several values of $N_{\mathrm{e}}=12.4,14.5,16.8,19\left[\times 10^{6}\right]$. The dependence of the equilibrium density $n_{\mathrm{eD}}$ on $V_{\mathrm{g}}$ was determined using the method from Fig. 2 , it is indicated by dotted lines. For each initial condition, we then applied a sequence of on/off microwave pulses and recorded the photocurrent $i_{\mathrm{pv}}$ induced by the electron redistribution. The measurement geometry is shown in $\mathbf{b}$. Two $i_{\mathrm{pv}}(t)$ traces are shown in $\mathbf{c}$ for two $V_{\mathrm{g}}$ values noted $V_{1}=4.83 \mathrm{~V}$ and $V_{2}=5.1 \mathrm{~V}$ at $N_{\mathrm{e}}=14.5 \times 10^{6}$. The change in the density $\delta n_{\mathrm{e}}$ due to irradiation was then determined from Equation 3 , it is proportional to the area below the $i_{\text {pv }}(t)$ curves (shaded region for $V_{g}=V_{1}$ ). Region $(I)$ shows a clear plateau at $n_{\mathrm{eM}} \simeq 3.5 \times 10^{6} \mathrm{~cm}^{-2}$, where the density is independent of the initial density distribution. (d) A magnification of region (II) shows the density in the guard under irradiation, determined approximatively from $n_{g M}=\left(N_{e}-n_{e M} S_{d}\right) / S_{g}$. 
between the two techniques is that during compressibility measurements microwaves are always present, maintaining the system in a non-equilibrium state, whereas in the photocurrent measurement the microwave on/off pulses continuously reset the system back to its equilibrium state. Thus, in the photocurrent measurements, the electrons must overcome an increasingly large energy barrier to reach the incompressible state as $V_{\mathrm{g}}$ increases. When the barrier becomes too large, the systems remains in its equilibrium state and the photocurrent vanishes abruptly. In contrast, in the compressibility measurement, the electron system remains in the incompressible state as $V_{\mathrm{g}}$ changes and the electrostatic barrier does not need to be overcome directly, allowing the incompressible state to exist over a wider parameter range. (In the Supplementary Figs 6-9, we provide a detailed comparison between the two measurement techniques and show that they are fully consistent once the described hysteretic behaviour is taken into account; additional experimental data is also provided in ref. 38 ).

The estimated charging energy must be provided by the microwave irradiation since it is the only energy source in our system, its amplitude corresponds to a surprisingly large number of photons absorbed per electron $\Delta_{\mathrm{e}} / \hbar \omega \simeq 170$, particularly in comparison with a two-level system that cannot absorb more than one photon. The energy of the absorbed photons thus needs to be transferred efficiently to other degrees of freedom. It could be transferred by the excitation of higher Landau levels. However, the theoretical calculations performed by Y. Monarkha to explain the origin of microwave-induced resistance oscillations for electrons on helium suggest that Landau levels higher than the photon energy are unlikely to be strongly populated ${ }^{39,40}$. These calculations give an accurate prediction for the phase of the resistance oscillations allowing us to exclude strong inter-Landau level excitation ${ }^{41}$.

The energy can also be accumulated by transitions within the same manifold of quasi-degenerate Landau levels as they are bent by the confinement potential. A possible mechanism for this absorption is provided by the negative conductivity models introduced theoretically to explain ZRS in heterostructures ${ }^{12}$. It has been predicted that a negative resistance state will stabilize through the formation of domains with a fixed built-in electric field $E_{\mathrm{c}}$. We performed simulations of the electron density profiles for an electric field dependent conductivity model with $\sigma(E) \propto\left(E^{2}-E_{\mathrm{c}}^{2}\right)$, which is believed to describe domain formation. Our simulations show that this model tends to fix the electron density gradients to generate the built-in field $E_{\text {c }}$. This changes the compressibility of the electron cloud depending on the number of stable domains in the system but without inducing an incompressible state. Thus, new theoretical developments are required to make the domain picture consistent with our experiments.

A recent theoretical proposal suggests that another instability can occur even before the conductivity becomes negative $e^{42,43}$. Due to fluctuations of the microwave electromagnetic field on the wavelength scale $\lambda \simeq 2 \mathrm{~mm}$ ratchet internal currents are expected to appear under irradiation inside the electron system. The velocity of the induced electron flow will scale as $v \sim \mu_{2} E_{\omega}^{2}$, where $E_{\omega}$ is the amplitude of the microwave electric field and $\mu_{2}$ a non-linear response coefficient ${ }^{43}$. Under steady-state conditions, this internal flow must be compensated by a counter-flow created by internal electric fields with maximal value $e n_{\mathrm{e}} / \epsilon_{0}$. The balance between the two flows sets a lower bound on the longitudinal mobility that is $\mu_{\mathrm{xx}}$ stable under irradiation: $\mu_{\mathrm{xx}} \sim \varepsilon_{0} \mu_{2} E_{\omega}^{2} / e^{2} n_{\mathrm{e}}$. Mobilities below this threshold are expected to lead to the formation of electron pockets on a scale $\lambda$ with a certain similarly to the stripe/puddles instability occurring in quantum Hall systems ${ }^{44}$.
The above argument predicts the existence of an instability without explaining the pinning of the electron density. A mechanism selecting a particular density value is thus desirable. As a possible mechanism, we propose the following scenario. If we assume that a significant fraction of the energy carried by the absorbed photons is transferred into ripplons with a typical wave number given by the inverse magnetic length $k_{r} \sim \sqrt{e B / \hbar}$ (ref. 45), then the helium surface will vibrate at frequency $\omega_{r} \sim\left(\gamma k_{r}^{3} / \rho\right)^{1 / 2}$, where $\gamma$ and $\rho$ are the helium surface tension and density, respectively ${ }^{28}$. Provided frequencies are matched, this vibration can interact resonantly with electronic modes. The frequency of the expected surface vibrations is $\omega_{r} \simeq 2 \pi \times 30 \mathrm{MHz}$ (for $B=0.79 \mathrm{~T}$ ) and has the same order of magnitude as the low wavelength magneto-shear modes ${ }^{46}$, which have frequency $\Omega_{s} \sim \frac{e^{2} n_{\mathrm{e}}^{3 / 2}}{\epsilon_{0} m \omega_{\mathrm{c}}}$. Equating these frequencies $\omega_{r} \sim \Omega_{s}$, we find an equation on the plateau density:

$$
n_{\mathrm{e}} \sim\left[\frac{\gamma}{\rho} \frac{\epsilon_{0}^{2} B^{2}}{e^{2}}\left(\frac{e B}{\hbar}\right)^{3 / 2}\right]^{1 / 3} \propto B^{7 / 6}
$$

For $B=0.79 \mathrm{~T}$, this formula gives the right order of magnitude for the plateau density $n_{e} \simeq 5.8 \times 10^{6} \mathrm{~cm}^{-2}$, and the predicted dependence on $B$ is consistent with our observations at different $J=\omega / \omega_{c}$ shown in Fig. 4. Even if the described mechanism requires further theoretical studies, it correctly captures the critical dependence on the electronic density observed in the experiments. For electrons on helium, the electronic density mainly controls the strength of electronelectron interactions and the key role played by this parameter indicates that the formation of incompressible states is a collective effect involving many electrons.

In conclusion, we have shown that electrons trapped on a helium surface exhibit incompressible behaviour under resonant irradiation conditions corresponding to the formation of a microwave-induced ZRS. Their density becomes pinned to a fixed value independent of the applied electrostatic force and on the initial electron distribution profile for a wide range of parameters. The transition to the incompressible state is achieved by overcoming an impressively high electrostatic energy barrier of up to $0.1 \mathrm{eV}$ per electron. We described the possible energy conversion processes within the electron system that can transform the energy of the absorbed photons into charging energy, and we proposed several competing mechanisms that can render the equilibrium density profile unstable. Since the incompressible behaviour emerging from our experiments is very elegant, we believe that its understanding will stimulate the emergence of new concepts for self-organization in quantum systems.

\section{Methods}

Our experiments were performed in a Leiden dilution refrigerator with base temperature around $25 \mathrm{mK}$, the magnetic field was provided by a homemade superconducting magnet with maximal field of $1 \mathrm{~T}$. The experimental cell was filled with liquid helium until half filling by monitoring the cell capacitance during helium condensation. Electrons were deposited on the helium surface by thermionic emission from a heated tungsten filament. Control of the total electron number $N_{\mathrm{e}}$ in the cloud was achieved by trapping an initially high concentration of electrons and then lowering the confinement voltages allowing excess electrons to escape. The obtained $N_{\mathrm{e}}$ value was determined from compressibility measurements in equilibrium. The frequency of the intersubband transition was tuned in resonance with the photon energy using the linear Starck shift induced by the electric field perpendicular to the helium surface, which was fixed during our compressibility and photocurrent experiments to keep the system at intersubband resonance. The current probe electrodes were grounded through Stanford Research (SR570) current amplifiers, while the potential of the other electrodes was set by Yokogawa DC voltage supplies, this ensure a stable d.c. voltage on all cell electrodes independently of the irradiation. Numerical simulations were performed by solving the Laplace equations for our cell geometry using a finite elements method ${ }^{47}$. 


\section{References}

1. Klitzing, K. v., Dorda, G. \& Pepper, M. New method for high-accuracy determination of the fine-structure constant based on quantized hall resistance. Phys. Rev. Lett. 45, 494-497 (1980).

2. Tsui, D. C., Stormer, H. L. \& Gossard, A. C. Two-dimensional magnetotransport in the extreme quantum limit. Phys. Rev. Lett. 48, 1559-1562 (1982).

3. Jain, J. Composite Fermions (Cambridge Univ. Press, 2007).

4. Novoselov, K. S. et al. Two-dimensional gas of massless Dirac fermions in graphene. Nature 438, 197-200 (2005).

5. Zhang, Y., Tan, Y. W., Stormer, H. L. \& Kim, P. Stormer and Philip Kim experimental observation of the quantum Hall effect and Berry's phase in graphene. Nature 438, 201-204 (2005).

6. Bolotin, K. I., Ghahari, F., Shulman, M. D., Stormer, H. L. \& Kim, P. Observation of the fractional quantum Hall effect in graphene. Nature 462, 196-199 (2009).

7. Ezawa, Zyun Francis Quantum Hall Effect: Field Theoretical Approach and Related Topics (World scientific, 2008).

8. Mani, R. G. et al. Zero-resistance states induced by electromagnetic-wave excitation in GaAs/AlGaAs heterostructures. Nature 420, 646-650 (2002).

9. Zudov, M. A., Du, R. R., Pfeiffer, L. N. \& West, K. W. Evidence for a new dissipationless effect in 2D electronic transport. Phys. Rev. Lett. 90, 046807 (2003).

10. Durst, A. C., Sachdev, S., Read, N. \& Girvin, S. M. Radiation-induced magnetoresistance oscillations in a 2D electron gas. Phys. Rev. Lett. 91, 086803 (2003).

11. Dmitriev, I. A., Mirlin, A. D. \& Polyakov, D. G. Cyclotron-resonance harmonics in the ac response of a $2 \mathrm{D}$ electron gas with smooth disorder. Phys. Rev. Lett. 91, 226802 (2003).

12. Dmitriev, I. A., Mirlin, A. D., Polyakov, D. G. \& Zudov, M. A. Nonequilibrium phenomena in high Landau levels. Rev. Mod. Phys. 84, 1709-1763 (2012).

13. Chepelianskii, A. D. \& Shepelyansky, D. L. Microwave stabilization of edge transport and zero-resistance states. Phys. Rev. B 80, 241308 (2009).

14. Levin, A. D., Momtaz, Z. S., Gusev, G. M. \& Bakarov, A. K. Microwave-induced nonlocal transport in a two-dimensional electron system. Phys. Rev. B 89, 161304 (2014).

15. Mikhailov, S. A. Theory of microwave-induced zero-resistance states in two-dimensional electron systems. Phys. Rev. B 83, 155303 (2011).

16. Zhirov, O. V., Chepelianskii, A. D. \& Shepelyansky, D. L. Towards synchronization theory of microwave-induced zero-resistance states. Phys. Rev. B 88, 035410 (2013)

17. Chepelianskii, A. D., Pikovsky, A. S. \& Shepelyansky, D. L. Synchronization, zero-resistance states and rotating Wigner crystal. Eur. Phys. J. B 60, 225-229 (2007).

18. Willett, R. L., Pfeiffer, L. N. \& West, K. W. Evidence for current-flow anomalies in the irradiated 2D electron system at small magnetic fields. Phys. Rev. Lett. 93, 026804 (2004).

19. Smet, J. H. et al. Circular-polarization-dependent study of the microwave photoconductivity in a two-dimensional electron system. Phys. Rev. Lett. 95, 116804 (2005).

20. Zudov, M. A., Du, R. R., Pfeiffer, L. N. \& West, K. W. Bichromatic microwave photoresistance of a two-dimensional electron system. Phys. Rev. Lett. 96, 236804 (2006).

21. Mani, R. G., Johnson, W. B., Umansky, V., Narayanamurti, V. \& Ploog, K. Phase study of oscillatory resistances in microwave-irradiated- and dark-GaAs/AlGaAs devices: Indications of an unfamiliar class of the integra quantum Hall effect. Phys. Rev. B 79, 205320 (2009).

22. Wiedmann, S., Gusev, G. M., Raichev, O. E., Bakarov, A. K. \& Portal, J. C. Microwave zero-resistance states in a bilayer electron system. Phys. Rev. Lett. 105, 026804 (2010).

23. Dorozhkin, S. I., Pfeiffer, L., West, K., von Klitzing, K. \& Smet, J. H. Random telegraph photosignals in a microwave-exposed two-dimensional electron system. Nat. Phys. 7, 336 (2011)

24. Mani, R. G., Ramanayaka, A. N. \& Wegscheider, W. Observation of linearpolarization-sensitivity in the microwave-radiation-induced magnetoresistance oscillations. Phys. Rev. B 84, 085308 (2011).

25. Konstantinov, D. \& Kono, K. Photon-induced vanishing of magnetoconductance in 2D electrons on liquid helium. Phys. Rev. Lett. 105, 226801 (2010).

26. Konstantinov, D. \& Kono, K. Novel radiation-induced magnetoresistance oscillations in a nondegenerate two-dimensional electron system on liquid helium. Phys. Rev. Lett. 103, 266808 (2009).

27. In Two-Dimensional Electron Systems on Helium and Other Cryogenic Substrates. (ed Andrei, E. Y.) (Kluwer Academic, 1997).

28. Monarkha, Y. \& Kono, K. Two-Dimensional Coulomb Liquids and Solids (Springer, 2004).

29. Konstantinov, D., Chepelianskii, A. D. \& Kono, K. Resonant photovoltaic effect in surface state electrons on liquid helium. J. Phys. Soc. Jpn. 81, 093601 (2012).
30. Platzman, R. M. \& Dykman, M. I. Quantum computing with electrons floating on liquid helium. Science 284, 1967-1969 (1999).

31. Wilen, L. \& Giannetta, R. Impedance methods for surface state electrons. J. Low Temp. Phys. 72, 353-369 (1988).

32. Kovdrya, Y. Z., Nikolayenko, V. A., Kirichek, O. I., Sokolov, S. S. \& Grigor'ev, V. N. Quantum transport of surface electrons over liquid helium in magnetic field. J. Low Temp. Phys. 91, 371-389 (1993).

33. Closa, F., Raphaël, E. \& Chepelianskii, A. D. Transport properties of overheated electrons trapped on the a helium surface. Eur. Phys. J. B 87, 1-9 (2014).

34. Tessmer, S. H., Glicofridis, P. I., Ashoori, R. C., Levitov, L. S. \& Melloch, M. R. Subsurface charge accumulation imaging of a quantum Hall liquid. Nature 392, 51-54 (1997)

35. Badrutdinov, A. O., Abdurakhimov, L. V. \& Konstantinov, D. Cyclotron resonant photoresponse of a multisubband two-dimensional electron system on liquid helium. Phys. Rev. B 90, 075305 (2014).

36. Dolgopolov, V. T., Shashkin, A. A., Zhitenev, N. B., Dorozhkin, S. I. \& von Klitzing, K. Quantum Hall effect in the absence of edge currents. Phys. Rev. B 46, 12560-12567 (1992).

37. Jeanneret, B. et al. Observation of the integer quantum Hall effect by magnetic coupling to a Corbino ring. Phys. Rev. B 51, 9752-9756 (1995).

38. Watanabe, M., Nasyedkin, K., Kono, K., Konstantinov, D. \& Chepelianskii, A. D. Additional experimental data is available online at https://hal.archives-ouvertes.fr/hal-01143216.

39. Monarkha, Y. P. Microwave-resonance-induced magnetooscillations and vanishing resistance states in multisubband two-dimensional electron systems. Low Temp. Phys. 37, 655-666 (2011).

40. Monarkha, Y. P. Coulombic effects on magnetoconductivity oscillations induced by microwave excitation in multisubband two-dimensional electron systems. Low Temp. Phys. 38, 451-458 (2012).

41. Konstantinov, D., Monarkha, Y. \& Kono, K. Effect of coulomb interaction on microwave-induced magnetoconductivity oscillations of surface electrons on liquid helium. Phys. Rev. Lett. 111, 266802 (2013).

42. Entin, M. V. \& Magarill, L. I. Photogalvanic current in a double quantum well. JETP Lett. 98, 38 (2013).

43. Entin, M. V. \& Magarill, L. I. Photogalvanic current in electron gas over a liquid helium surface. JETP Lett. 98, 816-822 (2014).

44. Koulakov, A. A., Fogler, M. M. \& Shklovskii, B. I. Charge density wave in two-dimensional electron liquid in weak magnetic field. Phys. Rev. Lett. 76, 499-502 (1996).

45. Lea, M. J. et al. Magnetoconductivity of two-dimensional electrons on liquid helium:experiments in the fluid phase. Phys. Rev. B 55, 16280-16292 (1997).

46. Golden, Kenneth I., Kalman, G. \& Wyns, Philippe Dielectric tensor and collective modes in a two-dimensional electron liquid in magnetic field. Phys. Rev. B 48, 8882-8889 (1993).

47. Hecht, F. New development in FreeFem ++ . J. Numer. Math. 20, 251-265 (2012).

\section{Acknowledgements}

We are thankful to $\mathrm{H}$. Bouchiat for fruitful discussions and acknowledge support from JSPS KAKENHI Grant Number 24000007. One of us, A.C. acknowledges support from the E. Oppenheimer fellowship and from St Catharine college in Cambridge. D.K. is supported by internal grant from Okinawa Institute of Science and Technology Graduate University.

\section{Author contributions}

All authors contributed to all aspects of this work.

\section{Additional information}

Supplementary Information accompanies this paper at http://www.nature.com/ naturecommunications

Competing financial interests: The authors declare no competing financial interests.

Reprints and permission information is available online at http://npg.nature.com/ reprintsandpermissions/

How to cite this article: Chepelianskii, A.D. et al. An incompressible state of a photoexcited electron gas. Nat. Commun. 6:7210 doi: 10.1038/ncomms8210 (2015).

This work is licensed under a Creative Commons Attribution 4.0 International License. The images or other third party material in this article are included in the article's Creative Commons license, unless indicated otherwise in the credit line; if the material is not included under the Creative Commons license, users will need to obtain permission from the license holder to reproduce the material. To view a copy of this license, visit http://creativecommons.org/licenses/by/4.0/ 